\title{
Reconfigurable Fault Tolerant Control for Spacecraft Based on Modified IMM Algorithm
}

\author{
Li Xiaoyun ${ }^{1}$, Du Wei ${ }^{2}$ \\ ${ }^{1}$ Beijing Institute of Spacecraft System Engineering, Beijing, China \\ ${ }^{2}$ Beijing Institute of Control Engineering, Beijing, China
}

\begin{abstract}
This paper proposes an integrated fault detection, diagnosis, and reconfigurable control method for attitude tracking of a spacecraft. A novel IMM algorithm, based on the unscented Kalman filter and an index related to the closed-loop system performance, is presented to detect and diagnose the faults. To achieve steady attitude tracking, the sliding mode variable structure controller is designed. When a fault is detected and isolated, the controller structure is reconfigured to compensate the faulty system to maintain the system performance. A simulation example evaluating the attitude tracking process is employed, which demonstrates the efficiency of the proposed approach.
\end{abstract}

\section{Introduction}

Modern engineering systems are becoming more and more sophisticated. The requirements for their reliability, availability, and security also grow significantly. In the spacecraft attitude control system, failures of its actuator or sensor may cause serious problems and need to be detected and isolated as soon and as accurately as possible. However, due to the limit of mass, cost and other factors, spacecraft cannot be configured enough measurement devices to detect faults. So the question of how to maximize the use of analytical redundancy to diagnose and isolate faults is urged to be solved in practical projects.

A fault tolerant control system is a control system that possesses the ability to accommodate system failures automatically, and to maintain overall system stability and acceptable performance. Generally speaking, an active fault tolerant control system compensates for faults either by selecting a precomputed controller or by synthesizing a new control strategy on-line. Reconfigurable fault tolerant control as an active fault tolerant control method, fault detection and diagnosis (FDD) is its prerequisite.

In this paper, FDD is carried out using interacting multiple-model (IMM) algorithm in that the system model sequence is assumed to be a Markov chain with transition probabilities, which has superiority in fault diagnosis and state estimation for system involving structural as well as parametric changes. IMM algorithm has been used in maneuvering target tracking [1], FDD of dynamic systems [2], and so on.

In the traditional IMM algorithm, the extended Kalman filter (EKF) is the estimation unit and based on linearization technique can only achieve first-order accuracy. While the unscented Kalman filter (UKF) is based on the unscented transform technique. The state variable is represented by a minimal set of carefully chosen sample points, which are able to approximate the posterior mean and covariance of the output variable with second-order accuracy [3, 4]. Further, it is not necessary to compute explicitly the Jacobians in the UKF.

In the meanwhile, in the traditional IMM algorithm, model probabilities are used to provide an indication of the model in effect at a given time. However, when a control action is introduced and combined with a multiple-model estimator to form a closed-loop system, the effect of the feedback will force the residuals from different models to be similar and this affects the discrimination property of the filter. Such a situation is even more serious in reconfigurable control design because the objective of a reconfigurable control is to make the performance of the reconfigured system as close to that of the prefault system as possible [5]. Inspired by the multiple-model switching and tuning approach, in this paper, a performance index is used to provide additional information for reliable fault diagnosis.

Furthermore, the attitude tracking controller of a sliding mode variable structure controller is designed. Once a fault is detected, the reconfigurable fault tolerant controller is adopted to accommodate the faulty system.

\section{Kinematics and dynamics models}

Taking into account spacecraft need large angle maneuver and bring singularity, the attitude kinematics equation is described by unit quaternion 


$$
\left\{\begin{array}{l}
\dot{q}_{0}=-\frac{1}{2} \boldsymbol{q}_{13}^{\mathrm{T}} \boldsymbol{\omega} \\
\dot{\boldsymbol{q}}_{13}=\frac{1}{2}\left(q_{0} \boldsymbol{I}_{3}+\boldsymbol{q}_{13}{ }^{\times}\right) \boldsymbol{\omega}
\end{array}\right.
$$

where $\boldsymbol{\omega}=\left[\omega_{x}, \omega_{y}, \omega_{z}\right]^{\mathrm{T}}$ is the angular velocity; $\left(q_{0}, \boldsymbol{q}_{13}\right) \in R \times R^{3}$ is the attitude quaternion satisfying $\boldsymbol{q}_{13}=\left[q_{1}, q_{2}, q_{3}\right]^{\mathrm{T}}$ and $q_{0}^{2}+\boldsymbol{q}_{13}^{\mathrm{T}} \boldsymbol{q}_{13}=1 ; \boldsymbol{I}_{3}$ denotes an $3 \times 3$ identity matrix; the notation $q_{13}{ }^{\times}$denote a skewsymmetric matrix.

Ignoring the flexibility and liquid sloshing, the attitude dynamics equitation can be described by a rigid body model as follows

$$
\boldsymbol{J} \dot{\boldsymbol{\omega}}+\boldsymbol{\omega}^{\times} \boldsymbol{J} \boldsymbol{\omega}=\boldsymbol{T}_{c}+\boldsymbol{T}_{d}
$$

where $\boldsymbol{J}$ is the inertia matrix of a spacecraft. $\boldsymbol{T}_{c}$ and $\boldsymbol{T}_{d}$ denote the vectors of control torques and disturbance torques respectively. $\left|\boldsymbol{T}_{d}(t)\right| \leq T_{d \max }, T_{d \max }>0$.

\section{FDD based on IMM algorithm}

Since faults system cannot be modelled well by a single set of equations of state that varies continuously. A more appropriate mathematical model for such a system is the so-called stochastic hybrid system. It differs from the conventional stochastic systems in that its state may jump as well as vary continuously.

Euler discretization from equations (1) and (2), the following model can be available

$$
\left\{\begin{aligned}
\boldsymbol{\omega}(k+1)= & T \boldsymbol{J}^{-1}(m(k+1))\left[\boldsymbol{T}_{c}(k)+\boldsymbol{T}_{d}(k)-\right. \\
& \left.\boldsymbol{\omega}(k)^{\times} \boldsymbol{J}(m(k+1)) \boldsymbol{\omega}(k)\right]+ \\
& \boldsymbol{\omega}(k)+\boldsymbol{\zeta}_{1}(k, m(k+1)) \\
q_{0}(k+1)= & -\frac{T}{2} \boldsymbol{q}_{13}^{\mathrm{T}}(k) \boldsymbol{\omega}(k)+q_{0}(k) \\
\boldsymbol{q}_{13}(k+1)= & \frac{T}{2}\left[q_{0}(k) \boldsymbol{I}_{3}+\boldsymbol{q}_{13}(k)^{\times}\right] \boldsymbol{\omega}(k)+\boldsymbol{q}_{13}(k)
\end{aligned}\right.
$$

where $T$ is the sampling interval; $m(k)$ is the discretevalued modal state at time $k ; \zeta_{1}$ is the discrete time process noises.

Supposed that state vector

$$
\boldsymbol{x}(k)=\left[\begin{array}{ccc}
\boldsymbol{\omega}^{\mathrm{T}}(k) & q_{0}(k) & \boldsymbol{q}_{13}^{\mathrm{T}}(k)
\end{array}\right]^{\mathrm{T}}
$$

measurement vector

$$
\boldsymbol{z}(k)=\boldsymbol{x}(k)+\boldsymbol{\eta}(k)
$$

where $\boldsymbol{\eta}(k)$ is the discrete time measurement noises.

Based on the above, the stochastic hybrid system of attitude control system is can be described as follows

$$
\left\{\begin{array}{c}
\boldsymbol{x}(k+1)=\boldsymbol{A}(k, m(k+1)) \boldsymbol{x}(k)+ \\
\boldsymbol{F}(k, m(k+1)) \boldsymbol{M}(k)+ \\
\boldsymbol{H}(k, m(k+1)) \boldsymbol{\zeta}(k, m(k+1)) \\
\boldsymbol{z}(k)=\boldsymbol{C}(k, m(k)) \boldsymbol{x}(k)+\boldsymbol{\eta}(k, m(k))
\end{array}\right.
$$

where $\boldsymbol{A}, \boldsymbol{F}, \boldsymbol{H}, \boldsymbol{C}$ are the corresponding dimension matrices; $\boldsymbol{M}$ is the sum of $\boldsymbol{T}_{c}$ and $\boldsymbol{T}_{d} . \boldsymbol{\zeta}$ and $\boldsymbol{\eta}$ are independent, representing system noises and measurement noises.

The system model sequence assumed to be a Markov chain with transition probabilities

$$
P\left\{m_{j}(k+1) \mid m_{i}(k)\right\}=\pi_{i j}, \forall m_{i}, m_{j} \in \boldsymbol{S}
$$

with

$$
\sum_{j} \pi_{i j}=1, \quad i, j=1,2, \cdots, N
$$

where $P\{\cdot\}$ denotes probability; $\pi_{i j}$ is the transition probability from the model $i$ to the model $j$; the event that $m_{j}$ is in effect at time $k$ is denoted as $m_{j}(k) \square\left\{m(k)=m_{j}\right\} ; \boldsymbol{S}=\left\{m_{1}, m_{2}, \cdots, m_{N}\right\}$ is the set of all possible system models.

A complete cycle of the IMM-based FDD scheme with UKF as its model-matched filter is described as follows

(1) interaction of the estimates

model probability predicting [5]:

$$
\mu_{j}(k+1 \mid k)=\sum_{i} \pi_{i j} \mu_{i}(k)
$$

probability mixing:

$$
\mu_{i \mid j}(k)=\pi_{i j} \mu_{i}(k) / \mu_{j}(k+1 \mid k)
$$

estimate mixing:

$$
\hat{x}_{j}^{0}(k \mid k)=\sum_{i} \hat{x}_{i}(k \mid k) \mu_{i \mid j}(k)
$$

covariance mixing:

$$
\begin{gathered}
\boldsymbol{P}_{j}^{0}(k \mid k)=\sum_{i}\left\{\boldsymbol{P}_{i}(k \mid k)+\left[\hat{x}_{j}^{0}(k \mid k)-\hat{x}_{i}(k \mid k)\right]\right. \\
\left.\left[\hat{x}_{j}^{0}(k \mid k)-\hat{x}_{i}(k \mid k)\right]^{\mathrm{T}}\right\} \mu_{i \mid j}(k)
\end{gathered}
$$

\section{(2) unscented Kalman filter}

initialization:

$$
\begin{gathered}
\boldsymbol{x}_{0}=\mathrm{E}[\boldsymbol{x}(0)], \\
\boldsymbol{P}_{0}=\mathrm{E}\left[\left(\boldsymbol{x}(0)-\boldsymbol{x}_{0}\right)\left(\boldsymbol{x}(0)-\boldsymbol{x}_{0}\right)^{\mathrm{T}}\right]
\end{gathered}
$$

sample points and their weights:

$$
\begin{gathered}
\chi_{0}=\hat{x}(k \mid k), \quad \omega_{0}=\kappa /\left(n_{x}+\kappa\right) \\
\chi_{i}=\hat{x}(k \mid k)+\left(\sqrt{\left(n_{x}+\kappa\right) \boldsymbol{P}_{j}(k \mid k)}\right)_{i}, \\
\omega_{i}=1 /\left[2\left(n_{x}+\kappa\right)\right], \quad i=1,2, \cdots, n_{x} \\
\chi_{i}=\hat{x}(k \mid k)-\left(\sqrt{\left(n_{x}+\kappa\right) \boldsymbol{P}_{j}(k \mid k)}\right)_{i}, \\
\omega_{i}=1 /\left[2\left(n_{x}+\kappa\right)\right], \quad i=n_{x}+1, \cdots, 2 n_{x}
\end{gathered}
$$


where $n_{x}$ is the dimension of state variable; $\kappa \in R$ satisfies $n_{x}+\kappa \neq 0 ;\left(\sqrt{\left(n_{x}+\kappa\right) \boldsymbol{P}_{j}(k \mid k)}\right)_{i} \quad$ is the $i$ th row of the matrix square root.

sample points through the nonlinear function:

$$
\xi_{i}(k+1 \mid k)=f_{j}\left(\chi_{i}\right)
$$

state variable and covariance predicting:

$$
\begin{gathered}
\hat{x}_{j}(k+1 \mid k)=\sum_{i=0}^{2 n_{x}} \omega_{i} \xi_{i}(k+1 \mid k) \\
\boldsymbol{P}_{j}(k+1 \mid k)=\sum_{i=0}^{2 n_{x}} \omega_{i}\left[\left(\xi_{i}(k+1 \mid k)-\hat{x}_{j}(k+1 \mid k)\right) .\right. \\
\left.\left(\xi_{i}(k+1 \mid k)-\hat{x}_{j}(k+1 \mid k)\right)^{\mathrm{T}}\right]+\boldsymbol{Q}_{j}(k+1)
\end{gathered}
$$

measurement derived from sample points predicting:

$$
\varsigma_{i}(k+1 \mid k)=C_{j}(k) \xi_{i}(k+1 \mid k)
$$

measurement and covariance predicting:

$$
\begin{gathered}
\hat{z}_{j}(k+1 \mid k)=\sum_{i=0}^{2 n_{x}} \omega_{i} \varsigma_{i}(k+1 \mid k) \\
\boldsymbol{S}_{j}(k+1)=\sum_{i=0}^{2 n_{x}} \omega_{i}\left[\left(\varsigma_{i}(k+1 \mid k)-\hat{z}_{j}(k+1 \mid k)\right) .\right. \\
\left.\left(\varsigma_{i}(k+1 \mid k)-\hat{z}_{j}(k+1 \mid k)\right)^{\mathrm{T}}\right]+\boldsymbol{R}_{j}(k+1)
\end{gathered}
$$

cross-covariance of measurement and state variable predicting:

$$
\begin{gathered}
\boldsymbol{P}_{x z}=\sum_{i=0}^{2 n_{x}} \omega_{i}\left[\left(\xi_{i}(k+1 \mid k)-\hat{x}_{j}(k+1 \mid k)\right) .\right. \\
\left.\left(\varsigma_{i}(k+1 \mid k)-\hat{z}_{j}(k+1 \mid k)\right)^{\mathrm{T}}\right]
\end{gathered}
$$

UKF gain:

$$
\boldsymbol{W}_{j}(k+1)=\boldsymbol{P}_{x z} \boldsymbol{S}_{j}^{-1}(k+1)
$$

state and covariance updating:

$$
\begin{gathered}
v_{j}(k+1)=z_{j}(k+1)-\hat{z}_{j}(k+1 \mid k) \\
\hat{x}_{j}(k+1 \mid k+1)=\hat{x}_{j}(k+1 \mid k)+ \\
\boldsymbol{W}_{j}(k+1) v_{j}(k+1) \\
\boldsymbol{P}_{j}(k+1 \mid k+1)=\boldsymbol{P}_{j}(k+1 \mid k)- \\
\boldsymbol{W}_{j}(k+1) \boldsymbol{S}_{j}(k+1) \boldsymbol{W}_{j}(k+1)^{\mathrm{T}}
\end{gathered}
$$

(3) model probability updating

likelihood function:

$$
L_{j}(k+1)=\frac{\exp \left[-\frac{1}{2} v_{j}(k+1)^{\mathrm{T}} \boldsymbol{S}_{j}^{-1}(k+1) v_{j}(k+1)\right]}{\sqrt{\left|(2 \pi) \boldsymbol{S}_{j}(k+1)\right|}}
$$

model probability:

$$
\mu_{j}(k+1)=\frac{\mu_{j}(k+1 \mid k) L_{j}(k+1)}{\sum_{j} \mu_{j}(k+1 \mid k) L_{j}(k+1)}
$$

\section{(4) FDD logic}

residual vector:

$$
\varepsilon_{j}(k+1)=\left\|\boldsymbol{z}(k+1)-C_{j}(k+1) \hat{x}_{j}(k+1 \mid k+1)\right\|_{2}(30)
$$

index:

$$
J_{j}(k+1)=c_{1} \varepsilon_{j}^{2}(k+1)+c_{2} \sum_{i=k-D+2}^{k} \varepsilon_{j}^{2}(i)
$$

where $c_{1} \geq 0$ and $c_{2} \geq 0$ are constants, and $D$ is a moving window.

FDD logic:

$$
h(t)=\arg \min J_{j}(k+1)
$$

where $h(t)$ denotes the model in effect at time $t$ and $t \in[k T+T, k T+2 T)$.

Moreover, the aggregated state estimate, which is to be used in the following attitude tracking controller, is obtained as a probability-weighted sum of the updated state estimate from all the filters in the IMM algorithm

$$
\hat{x}(k+1 \mid k+1)=\sum_{j} \hat{x}_{j}(k+1 \mid k+1) \mu_{j}(k+1)
$$

\section{Reconfigurable fault tolerant control scheme}

\subsection{Attitude tracking controller design}

It is assumed that $\left(\psi_{i}, \vartheta_{i}, \gamma_{i}\right)$ and $\left(\psi_{f}, \vartheta_{f}, \gamma_{f}\right)$ are the initial attitude angles and final attitude angles, and the corresponding unit quaternion are $\left(q_{i 0}, \boldsymbol{q}_{i 13}\right)$ and $\left(q_{f 0}, \boldsymbol{q}_{f 13}\right), \boldsymbol{q}_{i 13}=\left[q_{i 1}, q_{i 2}, q_{i 3}\right]^{\mathrm{T}}, \boldsymbol{q}_{f 13}=\left[q_{f 1}, q_{f 2}, q_{f 3}\right]^{\mathrm{T}}$.

To achieve the desired attitude tracking, the Lyapunov function is chose as follows $[6,7]$.

$$
V=\sum_{i=0}^{3}\left(q_{i}-q_{f i}\right)^{2}
$$

from(1) and (34),

$$
\begin{aligned}
& \dot{V}=\omega_{x}\left(q_{f 0} q_{1}-q_{f 1} q_{0}-q_{f 2} q_{3}+q_{f 3} q_{2}\right)+ \\
& \omega_{y}\left(q_{f 0} q_{2}-q_{f 1} q_{3}-q_{f 2} q_{0}+q_{f 3} q_{1}\right)+ \\
& \omega_{z}\left(q_{f 0} q_{3}-q_{f 1} q_{2}-q_{f 2} q_{1}+q_{f 3} q_{0}\right)
\end{aligned}
$$

to satisfy $\dot{V} \leq 0$, assuming the following switching function $\boldsymbol{s}=\left[s_{1}, s_{2}, s_{3}\right]^{T}$ and

$$
\left\{\begin{array}{l}
s_{1}=\omega_{x}+k_{1}\left(q_{f 0} q_{1}-q_{f 1} q_{0}-q_{f 2} q_{3}+q_{f 3} q_{2}\right) \\
s_{2}=\omega_{y}+k_{2}\left(q_{f 0} q_{2}-q_{f 1} q_{3}-q_{f 2} q_{0}+q_{f 3} q_{1}\right) \\
s_{3}=\omega_{z}+k_{3}\left(q_{f 0} q_{3}-q_{f 1} q_{2}-q_{f 2} q_{1}+q_{f 3} q_{0}\right)
\end{array}\right.
$$

where $k_{i}>0, i=1,2,3$. So $\boldsymbol{s}=0$ when the system come into the sliding mode area and $\dot{V} \leq 0$. the constant rate reaching law

$$
\dot{\boldsymbol{s}}=-s_{0} \operatorname{sgn}(\boldsymbol{s})-\lambda \boldsymbol{s}
$$

where $s_{0}>0, \lambda>0$.

To avoid chattering, sgn function in (37) is replaced by tanh function as follows

$$
\tanh \left(\frac{\boldsymbol{s}}{\varepsilon}\right)=\frac{\sinh (\boldsymbol{s} / \varepsilon)}{\cosh (\boldsymbol{s} / \varepsilon)}=\frac{e^{s / \varepsilon}-e^{-s / \varepsilon}}{e^{s / \varepsilon}+e^{-s / \varepsilon}}
$$


where $\varepsilon>0$ and

$$
\dot{s}=-s_{0} \tanh \left(\frac{s}{\varepsilon}\right)-\lambda s
$$

To achieve attitude tracking, the inequation $s^{\mathrm{T}} \dot{S}<0$ should come into existence, so the following controller can be obtained

$$
\begin{aligned}
& \boldsymbol{M}_{c}=\boldsymbol{\omega}^{\times} \boldsymbol{J} \boldsymbol{\omega}-\boldsymbol{M}_{d}-\frac{1}{2} \boldsymbol{K}(J) \boldsymbol{P}\left(q_{f}\right) \boldsymbol{G}(\omega) \boldsymbol{q}- \\
& \left.s_{0} \tanh \left(\frac{\boldsymbol{s}}{\varepsilon}\right)-\lambda \boldsymbol{s}\right]
\end{aligned}
$$

\subsection{Fault tolerant controller design}

In this paper, three modes are considered: normal mode, actuator fault and sensor fault mode. Actuator fault would be modeled by annihilating the appropriate column of the control input matrix $\boldsymbol{F}$, that is, the elements of the appropriate column of matrix $\boldsymbol{F}$ become zeros. Similarly, sensor fault would be modeled by annihilating the appropriate row of the output matrix $\boldsymbol{C}$.

In the normal mode, controller (40) is used. In the fault mode, the fault tolerant controller is described by the following mathematical expression based on the online FDD information.

$$
\boldsymbol{M}_{c}(t)=\boldsymbol{M}_{j^{*}}(t)
$$

where $j^{*}=h(t)$.

\section{Numerical simulation}

In this section, a numerical simulation example evaluating the attitude tracking process is employed to demonstrate the efficiency of the proposed approach. $J_{x}=1600 \mathrm{~kg} \cdot \mathrm{m}^{2} \quad, \quad J_{y}=2900 \mathrm{~kg} \cdot \mathrm{m}^{2}$ ， $J_{z}=2350 \mathrm{~kg} \cdot \mathrm{m}^{2} \quad, \quad\left(\psi_{i}, \vartheta_{i}, \gamma_{i}\right)=\left(18^{\circ},-85.8^{\circ}, 0^{\circ}\right)$, $\left(\psi_{f}, \vartheta_{f}, \gamma_{f}\right)=\left(85.8^{\circ}, 0^{\circ}, 0^{\circ}\right) \quad, \quad T=0.01 \mathrm{~s}$, $M_{d}=0.005 \sin \left(\omega_{0} t\right) \mathrm{Nm}, \quad k_{i}=0.1, \quad \omega_{0}=1 \mathrm{rad} / \mathrm{s}$, $\varepsilon=0.5, \lambda=1000$; initial parameters for UKF: $\hat{x}_{j}(0 \mid 0)=x(0), \boldsymbol{P}_{j}(0 \mid 0)=100 I_{7}, c_{i}=0.5$; process noises and measurement noises are mutually independent with zeros mean, and their covariances are $\boldsymbol{R}_{j}=10^{-6} I_{7}$ and $\quad \boldsymbol{Q}_{j}=\operatorname{diag}\left(10^{-8}, 10^{-8}, 10^{-8}, 0,0,0,0\right) \quad$ respectively. Transition probability matrix is used as follows. Based on the parameters, 50 Monte Carlo numerical simulations are employed respectively for three cases.

$$
\Pi=\left[\begin{array}{ll}
0.9983 & 0.0017 \\
0.0011 & 0.9989
\end{array}\right]
$$

\section{(1) Normal mode}

In this case, the system of spacecraft is normal and not any actuator or sensor fault. As can be seen from Fig. 1, the sliding mode variable structure controller can meet the attitude tracking requirement, which has demonstrated the effectiveness of the designed controller.
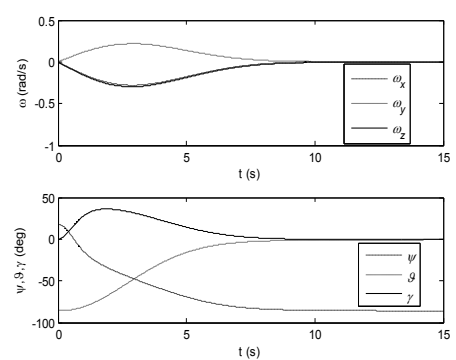

(a)
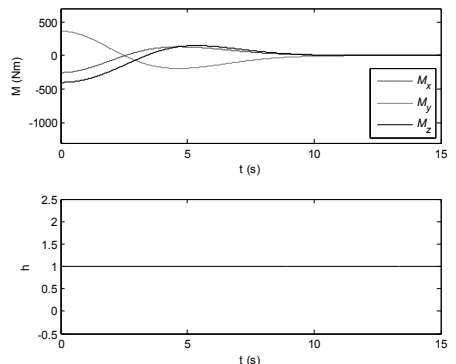

(b)

Figure 1. The curve of angle velocity, Euler angles, control moments and $h$ function (normal mode)

\section{(2) Actuator fault mode}

The process is divided into 2 models: from 0 second to 6 seconds, the system of spacecraft is normal; from 6 seconds to 15 seconds, the first component of the corresponding actuator is failure but sensors are not failure.
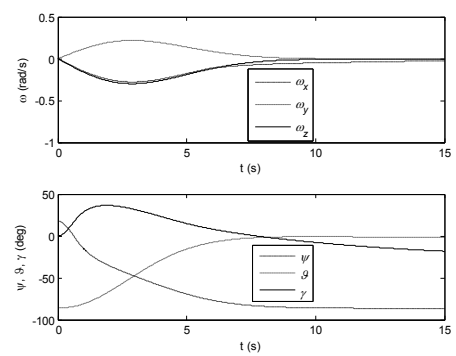

(a)
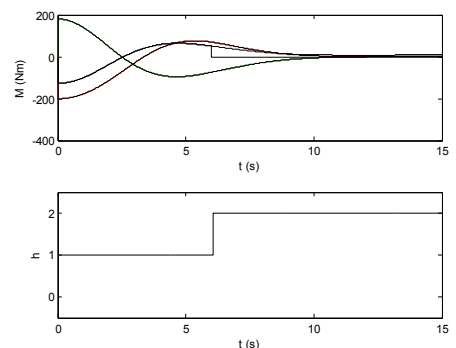

(b)

Figure 2. The curve of angle velocity, Euler angles, control moments and $h$ function (actuator faulty system without controller reconfiguration)

Table 1 The performance of proposed scheme (actuator fault)

\begin{tabular}{|c|c|c|c|c|c|}
\hline & CDID & FA & IFID & MFD & NMD \\
\hline Value & 0.9965333 & 0.00088 & 0 & 0.0025867 & 0 \\
\hline
\end{tabular}


In this table, CDID is correct detection and identification, FA is false alarm, IFID is incorrect fault identification, MFD is missed fault detection, NMD is no mode detection.
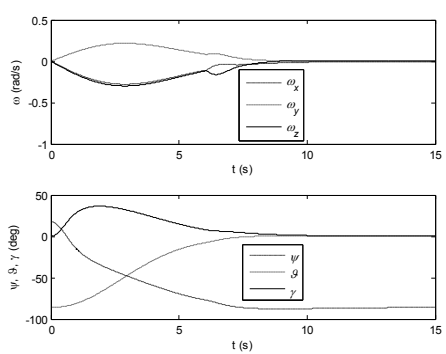

(a)
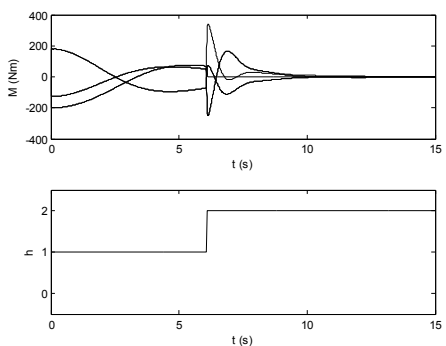

(b)

Figure 3. The curve of angle velocity, Euler angles, control moments and $h$ function (actuator faulty system with controller reconfiguration)

(3) Sensor fault mode

The process is divided into 2 models: from 0 second to 6 seconds, the system of spacecraft is normal; from 6 seconds to 15 seconds, the first component of the corresponding sensor is failure but actuators are not failure.
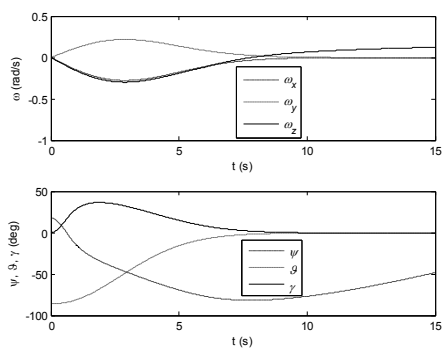

(a)
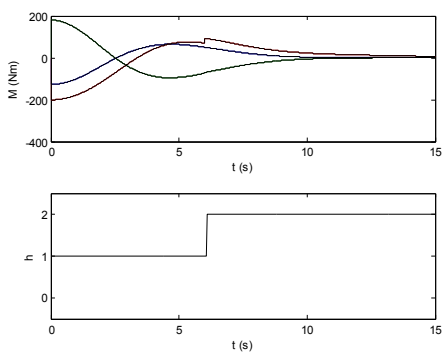

(b)

Figure 4. The curve of angle velocity, Euler angles, control moments and $h$ function (sensor faulty system without controller reconfiguration)

Table 2 The performance of proposed scheme (sensor fault)

\begin{tabular}{|l|l|l|l|l|l|}
\hline & CDID & FA & IFID & MFD & NMD \\
\hline
\end{tabular}

\begin{tabular}{|l|l|l|l|l|l|}
\hline Value & 0.9976667 & 0.001 & 0 & 0.0013333 & 0 \\
\hline
\end{tabular}
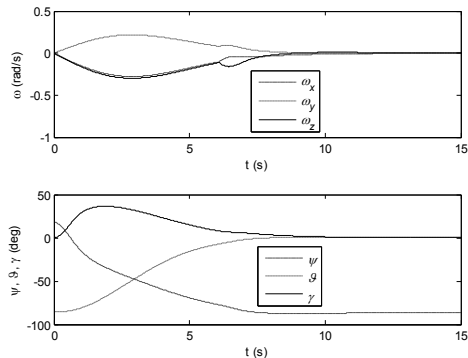

(a)
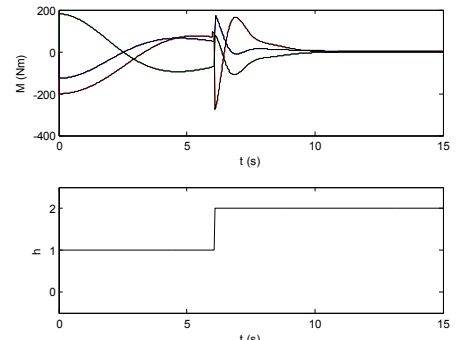

(b)

Figure 5. The curve of angle velocity, Euler angles, control moments and $h$ function (sensor faulty system with controller reconfiguration)

From the $h$ in the Fig. 2 to Fig. 5, and the table 1 and 2, the IMM algorithm with the UKF as the basic estimation unit and the output residual as the fault diagnosis index can detect and diagnose the fault condition in real time, and has high accuracy, low false alarm rate and false negative rate. Probability error is mainly caused by time delay.

In the case (2) and (3), the actuator or sensor is fault, but the faulty system is not reconstructed. From Fig. 2 and Fig. 4, there is a large attitude bias because the original designed controller for faulty system loses efficiency. However, based on the on-line FDD information, the real-time reconfiguration can be carried out. In Fig.3 and Fig.5, the sliding mode variable structure fault tolerant controller is adopted. Compared Fig.3, Fig.5 with Fig.2, Fig.4, the performance of the reconfigured system is very close to that of the normal system, which has tested the feasibility of reconfigurable fault tolerant method.

\section{Conclusion}

An integrated fault detection, diagnosis, and reconfigurable control scheme for a spacecraft with attitude tracking. FDD has been carried out using the modified IMM algorithm, based on the unscented Kalman filter and an index related to the closed-loop system performance. The sliding mode variable structure fault tolerance controller is designed based on this IMM algorithm. The Monte Carlo simulation results show that the IMM algorithm with the output residual as the fault diagnosis index can detect and diagnose the fault condition in real time. Steady attitude tracking has been achieved using fault tolerant controller is adopted to accommodate the faulty system to make the performance 
of reconfigured system to be close to that of the normal system. The simulation results have indicated that the proposed FDD and reconfigurable control scheme can deal with faults effectively. The disadvantage is that there is a certain delay in the diagnosis, how to design parameters more rationally and to improve the algorithm to reduce the delay time is the further content need to study.

\section{References}

1. Y.F. Han, Application of IMM Fusion Algorithm in Super-Low Altitude Target Detection [J], Systems Engineering and Electronics, 2003, 25(1): 15-17.

2. Y.M. Zhang, X.R. Li, Detection and Diagnosis of Sensor and Actuator Failures Using IMM Estimator [J], IEEE Transactions on Aerospace and Electronic Systems, 1998, 34(4): 1293-1313.

3. S. J. Julier, J. K. Uhlmann, H. F. Durrant-Whyte. A New Approach for the Nonlinear Transformation of
Means and Covariances in Filters and Estimators. IEEE Transactions on Automatic Control. 2000, 45(3): 477-482

4. K. Xiong, C.W. Chan, H.Y. Zhang, Detection of Satellite Attitude Sensor Faults Using the UKF[J], IEEE Transactions on Aerospace and Electronic System, 2007, 43(2): 480-491.

5. Y.M. Zhang, J. Jiang, Integrated Active FaultTolerant Control Using IMM Approach [J], IEEE Transactions on Aerospace and Electronic Systems, 2001, 37(4): 1221-1235.

6. K.M. MA, Z.Y. Dong, Variable Structure Attitude Control of Spacecraft Large Angle Maneuver Flight[J], Flight Dynamics, 2004, 22(2): 45-48.

7. L.J. Hu, F. C. Liu, Research on Attitude Maneuver Control of Large-ScaIe Satellite Based on Variable Structure Control System with Sliding Mode [J], Aerospace Shanghai, 2011, (1): 23-27. 